\section{The deficiency of Akt1 is sufficient to suppress tumor development in Pten $^{+/-}$mice}

\author{
Mei-Ling Chen, ${ }^{1,6}$ Pei-Zhang Xu, ${ }^{1,6}$ \\ Xiao-ding Peng, ${ }^{1}$ William S. Chen, ${ }^{1}$ \\ Grace Guzman, ${ }^{2}$ Ximing Yang, ${ }^{3}$ \\ Antonio Di Cristofano, ${ }^{4}$ Pier Paolo Pandolfi, ${ }^{5}$ \\ and Nissim Hay ${ }^{1,7}$
}

\begin{abstract}
${ }^{1}$ Department of Biochemistry and Molecular Genetics, University of Illinois at Chicago, Chicago, Illinois 60607, USA; ${ }^{2}$ Department of Pathology, University of Illinois at Chicago, Chicago, Illinois 60607, USA; ${ }^{3}$ Department of Pathology, Northwestern Medical School, Chicago, Illinois 60611, USA; ${ }^{4}$ Human Genetics Program, Fox Chase Cancer Center, Philadelphia, Pennsylvania 19111, USA; ${ }^{5}$ Cancer Biology and Genetics Program, Department of Pathology, Sloan-Kettering Institute, Memorial Sloan-Kettering Cancer Center, New York, New York 10021, USA
\end{abstract}

The tumor suppressor PTEN is frequently inactivated in human cancers. A major downstream effector of PTEN is Akt, which is hyperactivated via PTEN inactivation. It is not known, however, whether diminished Akt activity is sufficient to inhibit tumorigenesis initiated by Pten deficiency. Here we showed that the deficiency of Akt1 is sufficient to dramatically inhibit tumor development in $\boldsymbol{P t e n}^{+/-}$mice. Akt1 deficiency had a profound effect on endometrium and prostate neoplasia, two types of human cancer, in which PTEN is frequently mutated, and also affected thyroid and adrenal medulla tumors and intestinal polyps. Even haplodeficiency of Akt1 was sufficient to markedly attenuate the development of highgrade prostate intraepithelial neoplasia (PIN) and endometrial carcinoma. These results have significant implications for cancer therapy.

Supplemental material is available at http://www.genesdev.org.

Received November 23, 2005; revised version accepted April $18,2006$.

The tumor suppressor PTEN is a phospholipids phosphatase, which dephosphorylates the D3 position of the inositol ring of phosphoinositides, the products of phosphatidylinositol 3-kinase (PI3K). PTEN therefore negates the activity of PI3K and inhibits the activities of its downstream effectors. One major downstream effector of PI3K and PTEN is the serine/threonine kinase Akt, also known as protein kinase B (PKB). Mammalian cells contain three genes that encode three Akt isoforms (Akt1-

[Keywords: Cancer therapy; PIN; endometrium carcinoma; thyroid and adrenal tumors; intestinal polyps]

${ }^{6}$ These authors contributed equally to this work.

${ }^{7}$ Corresponding author.

E-MAIL nhay@uic.edu; FAX (312) 355-2032.

Article is online at http://www.genesdev.org/cgi/doi/10.1101/gad.1395006.
3). The cDNAs of the three genes have $>85 \%$ sequence identity, and their protein products share the same structural organization. All three Akt isoforms possess conserved threonine and serine residues (T308 and S473 in Akt1) that are critical for the full activation of Akt. All Akt isoforms seem to have identical or similar substrate specificity, and it is not clear whether they possess different functional specificities in vivo. (Kandel and Hay 1999; Brazil and Hemmings 2001).

PTEN is frequently inactivated in human cancers (Sansal and Sellers 2004). In particular, a high incidence of PTEN inactivation has been observed in prostate and endometrial cancers, glioblastoma, and melanoma. Thus, it is important to identify therapeutic strategies that counteract PTEN inactivation to inhibit cancer. One attractive therapeutic target is Akt. It is not known, however, whether inactivation of Akt is sufficient to alleviate the impact of PI3K activation mediated by PTEN deficiency, as other targets of PI3K, including Rho GTPases, Tec family kinases, and PDK1 as well as other AGC kinase family members (Cantley 2002; Wymann and Marone 2005), could also contribute to the genesis of cancer. Furthermore, because of the pleiotropic activities of Akt, it is not known whether ablating Akt activity to the extent needed to inhibit cancer will have severe physiological consequences. Here we used mouse genetics to address these questions.

Haplodeficiency of Pten, in mice, elicits a wide range of tumors with a high tumor incidence in prostate, endometrium, thyroid, adrenal medulla, and the intestine (Di Cristofano et al. 1998; Suzuki et al. 1998; Podsypanina et al. 1999). We therefore crossed Pten ${ }^{+/-}$ mice with $A k t 1^{-/-}$mice, which are not impaired in their lifespan, to examine the ability of Akt1 deficiency to inhibit tumor development in $\mathrm{Pten}^{+/-}$mice. We found that Akt1 deficiency is sufficient to inhibit prostate neoplasia, endometrial carcinoma, thyroid and adrenal medulla tumors, and intestinal polyps markedly in Pten $^{+/-}$mice, with the most profound effects in the prostate, endometrium, and small intestine. In these cases, even haplodeficiency of Akt1 was sufficient to inhibit tumor development significantly. Finally, we showed that Akt1 deficiency is sufficient to markedly reduce lymphoid hyperplasia in Pten $^{+/-}$mice. Thus, our results imply that it is possible to inhibit Akt activity partially to the extent that it could be used as an approach for cancer therapy without severe physiological consequences.

\section{Results and Discussion}

To determine whether partial ablation of Akt activity could inhibit tumor development induced by Pten deficiency without severe physiological consequences, we crossed Pten $^{+/-}$mice, which develop diverse types of tumors, with $A k t 1^{-/-}$mice, which do not show a decreased lifespan (and possibly increased lifespan) (Supplementary Fig. S1; data not shown) despite spontaneous apoptosis in certain organs (Chen et al. 2001). The resulting Pten ${ }^{+/-}$Akt1 $^{+/-}$mice were generated in the C57BL/6/ 129 sv (1:1) background. Pten ${ }^{+-} A k t 1^{+/-}$mice were inter crossed to generate Pten ${ }^{+/-}$, Pten $^{+/-} \mathrm{Akt}^{+/-}$, Pten $^{+/-} \mathrm{Akt1}^{-/-}$, and wild-type mice. 


\section{Akt1 deficiency is sufficient to inhibit the development of PIN in Pten heterozygous mice}

PTEN is frequently mutated in human prostate cancer (Sansal and Sellers 2004), and Pten ${ }^{+/-}$mice frequently develop prostate intraepithelial neoplasia (PIN) (Di Cristofano et al. 1998; Suzuki et al. 1998; Podsypanina et al. 1999). We therefore determined PIN development in the three prostate lobes of 11-mo-old $\mathrm{Pten}^{+/-}, \mathrm{Pten}^{+/-}$ $\mathrm{Akt1}^{+/-}$, and $\mathrm{Pten}^{+/-} \mathrm{Akt1}^{-/-}$mice using histopathological criteria as well as BrdU incorporation and K14 staining.

For the histopathological analysis, the grades of PIN were defined according to previously described criteria (Shappell et al. 2004) (Supplementary Fig. S2a). In the high-grade lesions (PIN3 and PIN4), we often found diminished PTEN staining and increased plasma membrane staining of Akt phosphorylated at Ser473 (pAkt) (Fig. 1A; Supplementary Fig. S2b), demonstrating reduced expression or loss of the wild-type Pten allele with concomitant activation of Akt in the lesions. The strong plasma membrane staining of pAkt was often correlated with strong PCNA staining (Supplementary Fig. S2c), indicating that cells in which Akt was activated were in a proliferative state. We monitored PIN3 and PIN4 and observed that all Pten ${ }^{+/-}$mice had PIN3 and PIN4 lesions in the anterior lobe $(-16 \%$ with PIN3 and $\sim 84 \%$ with PIN4 lesions) (Fig. 1B). Even haplodeficiency of Akt1 markedly reduced the frequency of PIN4 lesions in the anterior lobe to $\sim 29 \%$, although the number of mice with PIN3 lesions was increased to $50 \%$. We observed a dramatic reduction in PIN4 lesions in $\mathrm{Pten}^{+/-}$Akt1 ${ }^{-/-}$ mice, of which only $4 \%$ and $11 \%$ had PIN4 and PIN3 lesions, respectively. Similar results were found in the dorsolateral lobe (Fig. 1B). In the ventral lobe, $\sim 82 \%$ and $4 \%$ of Pten $^{+/-}$mice developed PIN4 and PIN3 lesions, respectively, whereas only $\sim 24 \%$ of $\mathrm{Pten}^{+/-} \mathrm{Akt}^{+/-}$mice developed PIN4 lesions and 18\% developed PIN3 lesions. Pten ${ }^{+/}$Akt1 $^{-/-}$mice did not develop PIN3 and PIN4 lesions at all in the ventral lobe (Fig. 1B), and $46 \%$ of these mice were completely free of lesions in the ventral lobe.

PIN3 and PIN4 lesions are also characterized by the presence of mitotic figures. We therefore analyzed proliferation in the prostate by measuring the level of BrdU incorporation. There was a significant increase $(P<0.01)$ in BrdU incorporation in all-prostate lobes of the $\mathrm{Pten}^{+/-}$ mice when compared with wild type (Fig. 1C). In contrast, BrdU incorporation in Pten $^{+/-} A k t 1^{-1-}$ mice was indistinguishable $(P>0.05)$ from that seen in wild-type mice.

It was previously reported that high grades PIN in Pten $^{+/-}$mice are characterized by increased expression of K14 in the lumen (Park et al. 2002). In the normal prostate, K14-positive cells were confined to the basal layer, whereas in high-grade PIN, there were many large K14positive cells in the lumen of the duct (Supplementary Fig. S2d). We therefore used the intense K14 staining in the lumen of the ducts as an independent criterion for neoplastic lesions in the mouse prostate. We found that $85 \%$ of $\mathrm{Pten}^{+/-}$mice tested had intense K14 staining in the lumen of the anterior lobe and $\sim 3 \%$ of $\mathrm{Pten}^{+/-}$mice tested had intense K14 staining in the lumen of the dorsolateral lobe. In contrast, no intense K14 staining was observed in Pten ${ }^{+/}$Akt1 $1^{-/-}$mice (Supplementary Fig. S2d). Thus the K14 staining recapitulates the histopathological grading. The strong K14 staining also indicates
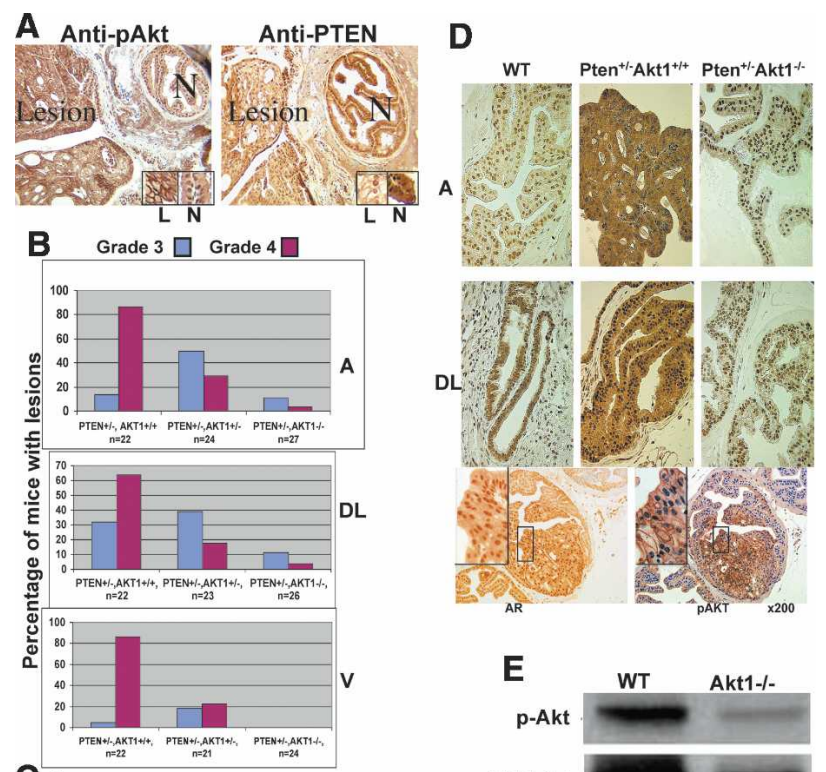

C
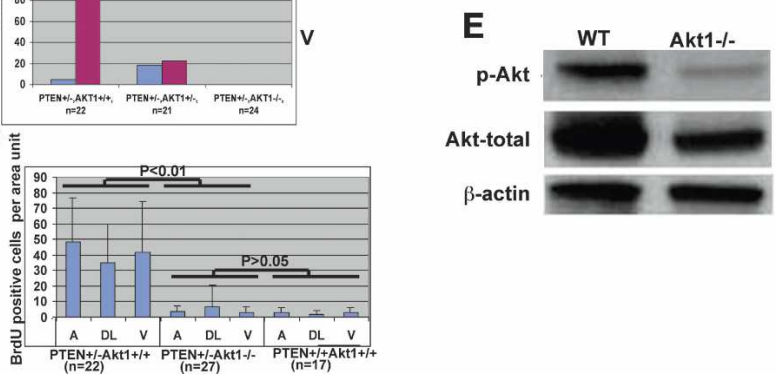

Figure 1. Aktl deficiency impairs the development of high-grade PIN in Pten ${ }^{+/-}$mice. (A) Paraffin-embedded anterior lobe sections derived from $\mathrm{Pten}^{+-}$mice were stained with either anti-pAkt or anti-PTEN. A PIN4 lesion (Lesion) and normal gland (N) are shown. (Insets) High magnification of an area for the lesion (L) or normal gland (N). (B) Incidence of PIN3 and PIN4 lesions in the three prostate lobes of Pten ${ }^{+/-}$, Pten ${ }^{+/-}$Akt1 $1^{+-}$, and Pten ${ }^{+-}$Akt1 ${ }^{-/-}$mice. The number of mice in each group is indicated. $(C)$ BrdU incorporation in the prostate lobes of Pten $^{+/}$, Pten $^{+/-} \mathrm{Akt1}^{+/-}$, and Pten ${ }^{+/-} \mathrm{Akt1}^{-/-}$ mice. The number of mice in each group is indicated in parentheses. BrdU-positive cells were counted as described in Materials and Methods. $P$ values were calculated for each prostate lobe in each genotype. $(D)$ Increased expression of androgen receptor in PIN4 lesions in the anterior and dorsolateral lobes correlates with increased pAkt in the lesions. (Top panels) Immunostaining with antiAR of paraffin-embedded sections derived form the anterior (A) and dorsolateral (DL) lobes of wild-type, Pten ${ }^{+-}$, and $\mathrm{Pten}^{+-} \mathrm{Akt1}^{-/}$ mice. (Bottom panel) Immunostaining with anti-AR or anti-pAkt of serial sections derived from the anterior lobe of Pten $^{+/-}$mice. (Insets) High magnifications. (E) Immunoblot of protein extracts derived from wild-type and $A \mathrm{kt1}^{-1-}$ mouse prostates using anti-pAkt, antipan-Akt, and anti- $\beta$-actin.

that the neoplastic cells may have originated from stem cells in the basal epithelium layer.

We also found that androgen receptor (AR) expression was dramatically elevated in the PIN4 lesions of Pten ${ }^{+/-}$ prostate as manifested by the strong staining with antiAR antibodies (Fig. 1D). Unlike AR localization in wildtype and $\mathrm{Pten}^{+-} \mathrm{Akt1}^{-1-}$ mice, AR was not confined only to the nucleus in Pten $^{+/-}$mice. Elevated AR expression was directly correlated with activation of Akt in the neoplastic lesions and was not present when Akt1 was deleted (Fig. 1D). Increased expression of AR in neoplastic cells supports the notion that these cells are of epithelial origin. These results also suggest that activation of Akt can eventually lead to elevation of AR expression and may explain why AR is overexpressed in human prostate tumors that do not contain AR gene amplification. The 
expression of AR in the neoplastic lesions suggests that they might be sensitive to hormone deprivation treatment. However, in light of the recent observation that elevated AR expression in human prostate tumors correlates with increased resistance to AR antagonists (Chen et al. 2004), these observations imply that prostate tumors in which Akt is activated may be more resistant to hormone therapy and that treatment of these tumors could benefit from the combination of Akt ablation and hormone therapy.

We then analyzed the activities of the two downstream effectors of Akt, FOXO and mTOR. The highgrade PIN lesions showed strong cytoplasmic staining for FOXO1 and strong staining for the phosphorylated S6 (pS6) ribosomal protein, which was used to indicate mTOR activity. FOXO1 staining was confined to nuclei in Pten $^{+/}{ }^{-} \mathrm{Akt}^{-/-}$prostate sections but was mostly cytoplasmic in Pten $^{+/-}$lesions (Supplementary Fig. S2e), indicating that it is phosphorylated and inactivated. S6 phosphorylation was dramatically reduced in $\mathrm{Pten}^{+/} A \mathrm{kt} 1^{-1-}$ prostate sections (Supplementary Fig. S2f), suggesting that mTOR activation as well as cytoplasmic localization of FOXO1 are associated with neoplasia and proliferation induced by the activation of Akt.

To determine the relative expression of the Akt isoforms in the different prostate lobes, we conducted RTPCR analysis of the three Akt genes: Akt1 was the major expressed isoform in all three prostate lobes (Supplementary Fig. S2g). Based on this analysis of mRNA expression, we estimate that Akt1 deficiency reduced total Akt activity by $\sim 50 \%$. This was further corroborated by the amounts of total and phosphorylated Akt protein in the prostates of $A k t 1^{-/-}$mice (Fig. 1E).

Taken together, these results demonstrate that the deficiency of $A k t 1$ alone is sufficient to inhibit prostate tumor development in Pten ${ }^{+/-}$mice and that even haplodeficiency of Akt1 can significantly attenuate the development of prostate neoplasia induced by Pten deficiency. As Pten deficiency has been observed in many primary human prostate cancer tumors as well as prostate cancer cell lines, these results should have an important impact on prostate cancer therapy.

\section{Akt1 deficiency inhibits development of endometrial carcinoma in $\mathrm{Pten}^{+/-}$mice}

Mutations in and deletions of PTEN are the most common genetic changes occurring in endometrial carcinomas (Ellenson and Wu 2004). Female Pten $^{+/-}$mice have a high incidence of endometrial neoplasia by 9 mo of age (Di Cristofano et al. 1998; Podsypanina et al. 1999). Female mice were sacrificed for analysis when they reached $40 \mathrm{wk}$ of age, and uterus sections were subjected to histopathological analyses. (The development of severe lymphoid hyperplasia in female $\mathrm{Pten}^{+/-}$mice ultimately leads to their death before $1 \mathrm{yr}$ of age [Di Cristofano et al. 1998; Podsypanina et al. 1999], precluding analysis of older female mice.)

Endometrial epithelial lesions were classified according to the following grades (Supplementary Fig. S3a): normal, simple hyperplasia (SH), mild atypical hyperplasia (AH1), moderate atypical hyperplasia (AH2), complex atypical hyperplasia (AH3), focal carcinoma in situ (CIS), and invasive carcinoma (CA).

In thirty-one 40-wk-old female $\mathrm{Pten}^{+/-}$mice examined, we found no mouse with a normal endometrium
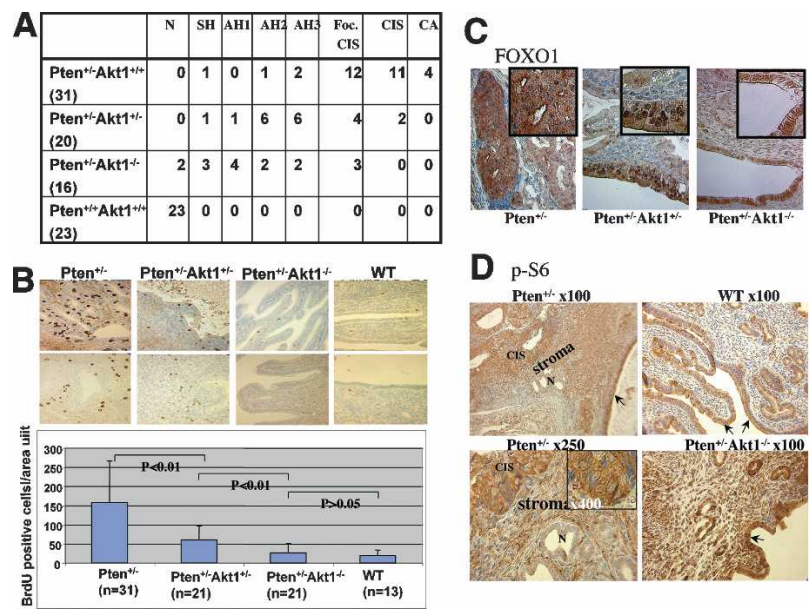

Figure 2. Akt1 deficiency is sufficient to inhibit the development of endometrial carcinoma in $\mathrm{Pten}^{+/-}$mice. (A) Incidence of endometrial neoplasia in Pten ${ }^{+/}$, Pten $^{+/-}$Akt1 $^{+/-}$, Pten $^{+/-} \mathrm{Akt1}^{-1-}$, and wildtype mice. Total number of mice examined in each group is indicated in parentheses. (B) BrdU incorporation in the uteri of Pten $^{+/}$, $\mathrm{Pten}^{+/-} \mathrm{Akt1}^{+-}{ }^{-}$, Pten $^{+-} \mathrm{Akt1}^{-/-}$, and wild-type mice. (Top panels) Representative areas of BrdU-positive cells. $(C)$ Localization of FOXO1 in the uteri of Pten $^{+/}$, Pten $^{+/} \mathrm{Akt1}^{+/}$, and $\mathrm{Pten}^{+-} \mathrm{Akt1}^{-/-}$ mice. Representative sections immunostained with anti-FOXO1, showing strong cytoplasmic staining in the lesion of $\mathrm{Pten}^{+-}$uteri, both cytoplasmic and nuclear staining in $\mathrm{Pten}^{+/-} \mathrm{Akt1}^{+/-}$uteri, and mostly nuclear staining in ten $^{+/-} A k t 1^{-/-}$uteri. (Insets) High magnifications. (D) Immunostaining with anti-pS6 of uterus sections derived from $\mathrm{Pten}^{+/-}$, wild-type, and $\mathrm{Pten}^{+/-} \mathrm{Akt1}^{-1-}$ mice. Representative sections showing strong staining of $\mathrm{pS} 6$ in the luminal epithelium and the lesions in Pten ${ }^{+-}$uteri $(100 \times, 250 x$, and 400x) that are confined only to the luminal epithelium (indicated by arrows) of wild-type and $\mathrm{Pten}^{+-} \mathrm{Akt1^{-/ }}$ uteri. CIS, stroma, and a normal gland (N) are indicated.

(Fig. 2A). In most cases (74\%), the highest-grade lesions were in the category of focal CIS or CIS, and in four mice $(-13 \%)$ the highest grade observed was invasive carcinoma. In contrast, in 20- to 40 -wk-old female Pten $^{+/-} A \mathrm{kt1}^{+/-}$mice examined, there were no invasive carcinomas, and focal CIS or CIS occurred in only six mice (33\%; Fig. 2A). Twelve Pten ${ }^{+/-} A k t 1^{+/-}$mice $(60 \%)$ had only low-grade lesions in the category of AH1 and AH2. The lesion grades were further reduced in Pten $^{+/-}$Akt1 ${ }^{-1-}$ mice (Fig. 2A). Two of 16 Pten $^{+/} A k t 1^{-/-}$ females $(12.5 \%)$ had a normal endometrium, and there were no mice with CIS or invasive carcinomas. In most of the Pten ${ }^{+/-} \mathrm{Akt1}^{-/-}$mice $(-69 \%)$, the endometrium displayed only low-grade lesions (simple hyperplasia to AH3). The highest-grade lesion was focal CIS, which was observed in only three mice $(-19 \%)$. Thus, the histopathological analyses clearly demonstrate that the reduction in Aktl is directly correlated with a marked attenuation in the genesis of endometrial neoplasia and progression to endometrial carcinoma.

To establish these results further, we analyzed BrdU incorporation in the uteri of female mice with the different genotypes (Fig. 2B). BrdU incorporation was markedly elevated in Pten $^{+/-}$mice when compared with wildtype mice. This elevated BrdU incorporation was significantly reduced in $\mathrm{Pten}^{+/-} \mathrm{Akt1^{+/- }}$ mice and was further diminished in Pten $^{+/-}$Akt1 ${ }^{-/-}$mice, as BrdU incorporation was observed mostly in CIS and invasive carcinoma.

We have quantified Akt activity in the endometrium using anti-phospho-Ser473 of Akt (Supplementary Fig. 
S3b) and found that $80 \%$ of the $\mathrm{Pten}^{+/-}$mice examined displayed activation Akt in the endometrium, whereas this was markedly reduced in $\mathrm{Pten}^{-1+} A k t 1^{+/-}$mice and further reduced in $\mathrm{Pten}^{+/} A \mathrm{At} 1^{-1-}$ mice. As described above for prostate lesions, we again analyzed the activities of the two downstream effectors of Akt, FOXO1 and $\mathrm{mTOR}$, in the uteri of mice with different genotypes. We found strong cytoplasmic staining of FOXO1 in highgrade lesions (AH3 and higher) (Fig. 2C; data not shown). Thus, in most lesions in Pten $^{+-}$uteri, we observed strong cytoplasmic FOXO1 staining. In most lesions in the uteri of $\mathrm{Pten}^{+/-} \mathrm{Akt1}^{+/-}$mice, we observed both cytoplasmic and nuclear staining of FOXO1 and, in most lesions in $\mathrm{Pten}^{+/} \mathrm{Akt1}^{-/-}$uteri, there was only nuclear staining of FOXO1 (Fig. 2C). Cytoplasmic staining for pS6 was confined to the luminal epithelium in wild-type mice (Fig. 2D). Strong cytoplasmic staining of pS6 was observed in high-grade lesions in $\mathrm{Pten}^{+/-}$mice, indicating hyperactivation of $\mathrm{mTOR}$ in the lesions. This strong cytoplasmic staining was diminished in $\mathrm{Pten}^{+/-} \mathrm{Akt1^{-/- }}$ uteri and was confined to the luminal epithelium as in wild-type mice, although we frequently observed nuclear pS6 staining in Pten $^{+/-}$Akt1 $1^{-/-}$uteri (Fig. 2D).

In summary, the absence of Aktl is sufficient to inhibit the development to endometrial carcinoma in Pten $^{+/-}$mice dramatically, and even haplodeficiency of Akt1 is sufficient to inhibit the development to endometrial carcinoma markedly. Akt1 is the most highly expressed Akt isoform in the uterus (Fig. 3E; Supplementary Fig. S5), and we estimate that the complete deletion of Akt1 could reduce total Akt activity in the endome-
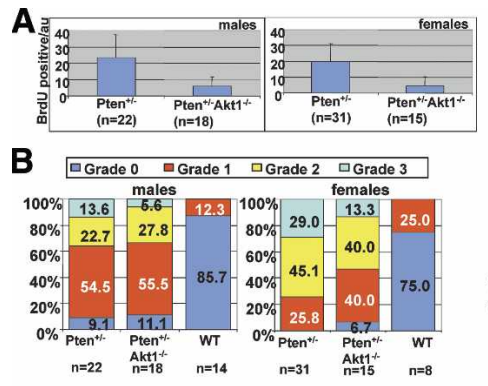

C

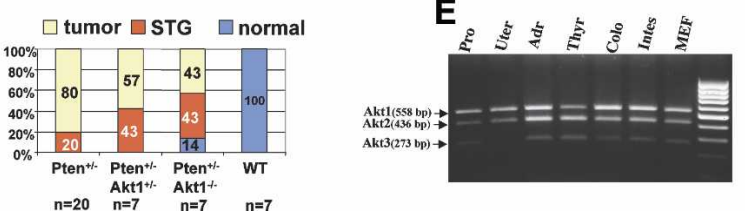

Figure 3. Effect of Akt1 deficiency on tumor development in the adrenal and thyroid glands and on the number of polyps in the small intestine of Pten ${ }^{+/-}$mice. (A) Quantification of BrdU incorporation in the adrenal medulla of Pten $^{+-}$and Pten ${ }^{+/-} \mathrm{Akt1}^{-/-}$11-mo-old male or 40-wk-old female mice. (B) Histopathological grades of the neoplastic lesions in adrenal medullas derived from $\mathrm{Pten}^{+/}$, Pten $^{+-} \mathrm{Akt1}^{-1-}$, and wild-type 11-mo-old male or 40-wk-old female mice. (C) Incidence of neoplastic lesions in thyroid glands derived from Pten $^{+-}$, Pten $^{+/-}$Akt1 1/-, Pten $^{+/-}$Akt1 $^{-/-}$, and wild-type 11mo-old male mice. $(D)$ The deficiency of Akt1 markedly reduced the number of polyps in the small intestine of $\mathrm{Pten}^{+/-}$mice. Quantification of the number of intestinal polyps in 11-mo-old male mice (top panel) or 40-wk-old female mice (bottom panel). The number of polyps \pm SD per mouse is shown. (E) Relative expression of Akt1,Akt2, and Akt3 mRNAs in prostate (lane 1), uterus (lane 2), adrenal gland (lane 3), thyroid (lane 4), colon (lane 5), intestine (lane 6), and in mouse embryo fibroblasts (lane 7). trium by $\sim 50 \%$. Therefore, the Akt1 haplodeficiency would reduce Akt activity in the endometrium by only $\sim 25 \%$. Thus, even a relatively small reduction in total Akt activity substantially reduces the progression to endometrial carcinoma in Pten $^{+/-}$mice. As PTEN mutations are the most common genetic changes occurring in human endometrial carcinoma (Ellenson and Wu 2004), even partial inhibition of Akt activity by small-molecule inhibitors could be used as an efficient therapy for endometrial carcinoma.

Interestingly, we observed a significant decrease in estrogen receptor $\alpha(\mathrm{ER} \alpha)$ nuclear staining in high-grade lesions and carcinoma (Supplementary Fig. S3c). In wildtype mice, strong nuclear staining of ER $\alpha$ was observed only in the glands that were in the proliferative phase (Supplementary Fig. S3c, panel A), whereas this was not observed in the secretory glands (Supplementary Fig. S3c, panel B). During tumor progression, however, we observed that $\mathrm{ER} \alpha$ expression is gradually diminished in the neoplastic cells (Supplementary Fig. S3c, panels $\mathrm{C}, \mathrm{D})$. This diminished nuclear staining of ER $\alpha$ was reciprocally correlated with the activation of Akt as measured by pAkt and pS6 staining (Supplementary Fig. S3d). Because proliferation of epithelial cells in the endometrium is dependent on estrogen receptor activity, these results suggest that Pten deficiency allows neoplastic cells to proliferate independently of estrogen receptor. This has implications for hormonal therapy of endometrial cancer exhibiting Pten deficiency.

The effect of Akt1 deficiency on the development of adrenal medullary and thyroid neoplasia, intestinal polyps, and lymphoid hyperplasia in $\mathrm{Pten}^{+/-}$mice

Consistent with previous results (Di Cristofano et al. 1998; Podsypanina et al. 1999|, we found that most male Pten $^{+/-}$mice sacrificed at $11 \mathrm{mo}$ of age and all female Pten $^{+-}$mice sacrificed at 40 wk of age developed bilateral adrenal medullary tumors. The development of adrenal neoplasia was accelerated in female mice when compared with male mice. The size of the adrenal medulla, which was directly correlated with the size of the tumors in Pten $^{+/-}$mice, was significantly decreased in Pten $^{+/-}$Akt1 $^{-/-}$mice (Supplementary Fig. S4a,b). In addition, the adrenal medulla tumors in Pten $^{+/-}$mice had high numbers mitotic figures; these were significantly reduced in Pten $^{+/}$Akt1 ${ }^{-1-}$ mice (data not shown). This was further corroborated by the analysis of BrdU incorporation (Fig. 3A).

In an attempt to categorize the neoplastic lesions observed in the histological analysis of the adrenal medulla, we utilized the grading criteria shown in Supplementary Figure $S 4 b$, whereas normal gland is a grade 0 and neoplastic glands were in the categories of grades 1-3. Mitotic figures and BrdU-positive cells were observed almost exclusively in grade 3 lesions. The percentage of male mice displaying grade 1 lesions was similar in both Pten ${ }^{+-}$and Pten ${ }^{+/-}$Akt1 $1^{-/-}$mice (Fig. 3B). While the percentage of $\mathrm{Pten}^{+/-} A k t 1^{-/-}$mice displaying grade 2 lesions was slightly higher than in $P_{t e n}{ }^{+/-}$mice, the percentage of male Pten ${ }^{+-} A k t 1^{-/-}$mice that had grade 3 lesions was $>50 \%$ less than what was observed for male $\mathrm{Pten}^{+/-}$mice. The number of female Pten ${ }^{+/}$ mice that had grade 2 and 3 lesions was markedly higher than that seen in male mice. The percentage of female Pten ${ }^{+-} \mathrm{Akt1}^{-/-}$mice that had grade 2 lesions was 
similar to that observed in female Pten $^{+/-}$mice, whereas the percentage of female $P t e n^{+/-} A k t 1^{-/-}$mice with grade 1 lesions was markedly higher, and, as was observed for male mice, female Pten ${ }^{+/-} A k t 1^{-1-}$ mice had a markedly reduced incidence of grade 3 lesions (Fig. 3B). Thus, although a complete ablation of $A k t 1$ did not significantly reduce the total incidence of neoplastic lesions in the adrenal medulla of $\mathrm{Pten}^{+/-}$mice, it did markedly reduce the incidence of high-grade lesions. Haplodeficiency of Akt1 did not reduce the development of neoplasia in the adrenal medulla in Pten $^{+/-}$mice, because we did not observe a reduction in the incidence and grades of neoplasia in Pten $^{+/-}$Akt1 ${ }^{+/-}$(data not shown). We also did not find a significant decrease in the size of the adrenal medulla (Supplementary Fig. S4a,b), mitotic figures, or BrdU incorporation in Pten ${ }^{+/}$Akt1 ${ }^{+/-}$mice (data not shown). Overall, the deficiency of Akt1 did not reduce tumorigenesis in the adrenal medulla to the same extent as was observed in the prostate and the endometrium. This could be attributed to the relatively lower level of Akt1 expression (relative to Akt2) in the adrenal medulla, when compared with their relative expression in the prostate and uterus (Fig. 3E; Supplementary Fig. S5). Alternatively, other downstream effectors of PI3K could be more critical than Akt for the development of adrenal medulla tumors.

Pten $^{+/-}$mice have a high frequency of neoplastic lesions in the thyroid glands (Di Cristofano et al. 1998; Podsypanina et al. 1999). We therefore analyzed the effect of Akt1 deficiency on the development of thyroid tumors in Pten ${ }^{+/-}$mice. All Pten ${ }^{+/-}$mice developed neoplastic lesions in the thyroid (Fig. 3C). There are two different degrees of lesions. Transformed glands that tend to blend into the surrounding normal-looking thyroid follicles were defined as separated transformed glands (STG), to distinguish them from aggregated transformed glands with nodule formation, which, based on their morphology, are consistent with thyroid adenomatoid nodules or multiple adenomas. These latter lesions were defined here simply as tumors. We examined thyroid lesions in 11-mo-old male mice. In Pten ${ }^{+/-}$mice, $80 \%$ developed thyroid tumors and only $20 \%$ had STG (Fig. 3C). There was a gradual decrease in the percentage of mice with thyroid tumors, which was correlated with reduced $A k t 1$ expression. In contrast, the percentage of mice with lower-grade lesions (STG) was increased when $A k t 1$ level was reduced. When Akt1 was completely deleted, the incidence of thyroid tumors was decreased by about twofold, and $\sim 14 \%$ of mice were free of any neoplastic lesions in the thyroid (Fig. 3C). Thus, Akt1 deficiency significantly reduced the incidence of thyroid tumors in Pten ${ }^{+/-}$mice, despite the localized lower level of Akt1 expression relative to Akt2 (Fig. 3E; Supplementary Fig. S5).

As was previously reported (Di Cristofano et al. 1998; Podsypanina et al. 1999), we found that Pten $^{+/-}$mice had a relatively high number of polyps in the small intestine. These polyps seemed to be adenomatous polyps (tubular adenoma) or hamartomatous polyps, and no invasive lesions could be detected. We monitored the mice for incidence and number of polyps in the small intestine. Almost $80 \%$ of 11 -mo-old male mice had polyps, and slightly more than $90 \%$ of 40 -wk-old females had polyps in the small intestine. The incidence of polyps was reduced in both males and females Pten $^{+/-} A k t 1^{-/-}$mice to $\sim 60 \%$. The number of polyps per mouse was substantially decreased in both males and females Pten $^{+/-} A k t 1^{-/-}$ (Fig. 3D). Interestingly, even haplodeficiency of $A k t 1$ was sufficient to reduce the percentage of polyp-positive mice almost to the same extent as was observed with complete $A k t 1$ deficiency as well as the average number of polyps (Fig. 3D). Because 11-mo-old male mice spontaneously develop polyps in the small intestine (Fig. 3D), the decrease in the incidence and number of polyps observed when Akt1 was deleted was more substantial than that observed in female mice (Fig. 3D). As estimated from the RT-PCR analysis (Fig. 3E; Supplementary Fig. S5), Akt1 is the major Akt isoform expressed in the small intestine and may explain the marked reduction in the number of polyps observed when Akt1 was deleted.

Because Pten $^{+-}$mice develop lymphoid hyperplasia and expansions of both $B$ and $T$ cell populations, we examined the effect of Aktl deficiency on the proliferation in lymph nodes (Supplementary Fig. S6a) and spleen (Supplementary Fig. S6b). In agreement with previous observations (Podsypanina et al. 1999), we found an increase in BrdU incorporation in the lymph nodes and spleen of $\mathrm{Pten}^{+/-}$and this was diminished in $P_{t e n}{ }^{+-} A k t 1^{-1-}$ mice to a level, which was not significantly different than in wild-type mice.

Finally, we found that $\sim 32 \%$ of female $\mathrm{Pten}^{+/-}$mice examined (Supplementary Fig. S6c) had nodules composed of tropoblasts, which resemble placental-site tropoblastic tumors (PSTT). This was almost completely diminished in Pten ${ }^{+/-}$Akt1 $^{-/-}$mice (Supplementary Fig. S6c). In addition, we found that $\sim 19 \%$ of female Pten $^{+1}$ mice developed benign and malignant adenomyoepithelioma in the mammary glands (Supplementary Fig. S6d,e). These tumors, which were found in multiple mammary glands (up to seven large tumors in one mouse) and in multiple foci, are composed of luminal epithelium and large number of spindle and polygonal myoephitelial cells as they were stained with both anti-K14 and anti-smooth muscles antigen (SMA) (Supplementary Fig. S6d). These tumors were completely eliminated in Pten $^{+/} \mathrm{Akt1}^{-/-}$mice (Supplementary Fig. S6e).

\section{Concluding remarks}

Here we have used a mouse model system to demonstrate that Akt1 deficiency, which does not shorten the lifespan of mice, can markedly decrease the incidence and development of tumors in Pten ${ }^{+/-}$mice in all tissues tested, with the most dramatic inhibition occurring in the prostate, endometrium, and small intestine. In these cases, even haplodeficiency of $A k t 1$ was able to reduce tumorigenesis. In the prostate, endometrium, and the intestine, Akt1 was the predominantly expressed Akt isoform, and its deficiency reduced total Akt activity by $\sim 50 \%$. Thus, haplodeficiency of Akt1 could reduce total Akt activity by only $\sim 25 \%$. Even a small reduction in total Akt activity was therefore sufficient to inhibit the development of tumors induced by PTEN inactivation. Furthermore, even in the adrenal medulla and (particularly) thyroid, where Akt1 was not the predominantly expressed isoform and Akt2 is expressed to the same or higher level of Akt1 (Fig. 3E; Supplementary Fig. S5), the reduction in tumorigenesis mediated by Akt1 deficiency was still effective. Thus, our results show that a deficiency of $A k t 1$ was most effective as an inhibitor of neo- 
plasia induced by PTEN inactivation. We cannot exclude the possibility, however, that Akt1 has a higher affinity than Akt2 for certain protein targets that affect tumorigenesis. It therefore remains to be determined whether Akt2 deficiency is more effective than Akt1 deficiency in the inhibition of adrenal medulla and thyroid tumors in Pten $^{+/-}$mice.

Since Akt1 was ablated prior to the development of tumors in $\mathrm{PTEN}^{+/-}$mice, it does not completely recapitulate potential therapy to ablate Akt activity after the development of tumors. Further studies using conditional Akt1 knockout mice are required to address this issue. Nevertheless our results have important implications for cancer therapy, as they indicate that partial inhibition of Akt activity or inhibition of individual Akt isoforms could be used for cancer therapy without severe side effects. Although the three Akt isoforms are highly homologous, which makes it difficult to design specific inhibitors for the individual isoforms, it was recently reported that such specificity can be obtained using small molecule inhibitors (DeFeo-Jones et al. 2005; Lindsley et al. 2005).

Akt is very frequently activated in human cancer not only via inactivation of PTEN, which occurs at a high frequency but also through activation of Ras, amplification and activation of the catalytic subunit of PI3K, and activation of growth factor receptors (Hay 2005). Thus, Akt is activated in a large number of human cancers, and therefore using partial ablation of Akt activity as a therapeutic approach may be not limited to tumors in which PTEN is inactivated.

\section{Materials and methods}

Source of mice

The Pten ${ }^{+-}$mice and $A k t 1^{-/-}$mice used in these studies were previously described (Di Cristofano et al. 1998; Chen et al. 2001).

Histology and immunocytochemistry

Histology and immunocytochemster were done as previously described (Peng et al. 2003). For details, see Supplemental Material.

Immunofluorescence staining, laser scanning confocal microscopy, immunoblotting, and RT-PCR analysis

See Supplemental Material.

BrdU incorporation assay

Mice were given i.p. injections of $0.5 \mathrm{mg}$ of BrdU per $10 \mathrm{~g}$ of body weight for $1 \mathrm{~h}$ before sacrificing. Tissues were collected and processed as described above. After dewaxing and rehydration, paraffin sections were digested by pepsin followed by digestion with EcoRI and Exonuclease III. Slides were then incubated with anti-BrdU and processed for immunohistochemistry. BrdU-labeled cells were counted from five fields at 250x magnifications for prostate and uterus and 400x magnifications for adrenal medulla. All counts were taken from the highest-labeled area of all sections.

Data analysis Data were entered using Microsoft Excel spreadsheets. $P$ values were calculated with a Student's $t$-test. Error bars represent the standard deviation.

\section{Acknowledgments}

This work was supported by grants from the NIH (CA090764 and AG016927) and in part by CapCURE to N.H.

\section{References}

Brazil, D.P. and Hemmings, B.A. 2001. Ten years of protein kinase B signalling: A hard Akt to follow. Trends Biochem. Sci. 26: 657-664. Cantley, L.C. 2002. The phosphoinositide 3-kinase pathway. Science
296: $1655-1657$.

Chen, W.S., Xu, P.Z., Gottlob, K., Chen, M.L., Sokol, K., Shiyanova, T., Roninson, I., Weng, W., Suzuki, R., Tobe, K., et al. 2001. Growth retardation and increased apoptosis in mice with homozygous disruption of the akt1 gene. Genes \& Dev. 15: 2203-2208.

Chen, C.D., Welsbie, D.S., Tran, C., Baek, S.H., Chen, R., Vessella, R., Rosenfeld, M.G., and Sawyers, C.L. 2004. Molecular determinants of resistance to antiandrogen therapy. Nat. Med. 10: 33-39.

DeFeo-Jones, D., Barnett, S.F., Fu, S., Hancock, P.J., Haskell, K.M., Leander, K.R., McAvoy, E., Robinson, R.G., Duggan, M.E., Lindsley, C.W., et al. 2005. Tumor cell sensitization to apoptotic stimuli by selective inhibition of specific Akt/PKB family members. Mol. Cancer Ther. 4: 271-279.

Di Cristofano, A., Pesce, B., Cordon-Cardo, C., and Pandolfi, P.P. 1998. Pten is essential for embryonic development and tumour suppression. Nat. Genet. 19: 348-355.

Ellenson, L.H. and Wu, T.C. 2004. Focus on endometrial and cervical cancer. Cancer Cell 5: 533-538.

Hay, N. 2005. The Akt-mTOR tango and its relevance to cancer. Cancer Cell 8: 179-183.

Kandel, E.S. and Hay, N. 1999. The regulation and activities of the multifunctional serine/threonine kinase Akt/PKB. Exp. Cell Res. 253: 210-229.

Lindsley, C.W., Zhao, Z., Leister, W.H., Robinson, R.G., Barnett, S.F., Defeo-Jones, D., Jones, R.E., Hartman, G.D., Huff, J.R., Huber, H.E., et al. 2005. Allosteric Akt (PKB) inhibitors: Discovery and SAR of isozyme selective inhibitors. Bioorg. Med. Chem. Lett. 15: 761-764.

Park, J.H., Walls, J.E., Galvez, J.J., Kim, M., Abate-Shen, C., Shen, M.M., and Cardiff, R.D. 2002. Prostatic intraepithelial neoplasia in genetically engineered mice. Am. J. Pathol. 161: 727-735.

Peng, X.D., Xu, P.Z., Chen, M.L., Hahn-Windgassen, A., Skeen, J., Jacobs, J., Sundararajan, D., Chen, W.S., Crawford, S.E., Coleman, K.G., et al. 2003. Dwarfism, impaired skin development, skeletal muscle atrophy, delayed bone development, and impeded adipogenesis in mice lacking Akt1 and Akt2. Genes \& Dev. 17: 1352-1365.

Podsypanina, K., Ellenson, L.H., Nemes, A., Gu, J., Tamura, M., Yamada, K.M., Cordon-Cardo, C., Catoretti, G., Fisher, P.E., and Parsons, R. 1999. Mutation of Pten/Mmac1 in mice causes neoplasia in multiple organ systems. Proc. Natl. Acad. Sci. 96: 1563-1568.

Sansal, I. and Sellers, W.R. 2004. The biology and clinical relevance of the PTEN tumor suppressor pathway. J. Clin. Oncol. 22: 2954-2963.

Shappell, S.B., Thomas, G.V., Roberts, R.L., Herbert, R., Ittmann, M.M., Rubin, M.A., Humphrey, P.A., Sundberg, J.P., Rozengurt, N., Barrios, R., et al. 2004. Prostate pathology of genetically engineered mice: Definitions and classification. The consensus report from the Bar Harbor meeting of the Mouse Models of Human Cancer Consortium Prostate Pathology Committee. Cancer Res. 64: 2270-2305.

Suzuki, A., de la Pompa, J.L., Stambolic, V., Elia, A.J., Sasaki, T., del Barco Barrantes, I., Ho, A., Wakeham, A., Itie, A., Khoo, W., et al. 1998. High cancer susceptibility and embryonic lethality associated with mutation of the PTEN tumor suppressor gene in mice. Curr. Biol. 8: 1169-1178.

Wymann, M.P. and Marone, R. 2005. Phosphoinositide 3-kinase in disease: Timing, location, and scaffolding. Curr. Opin. Cell Biol. 17: 141-149. 


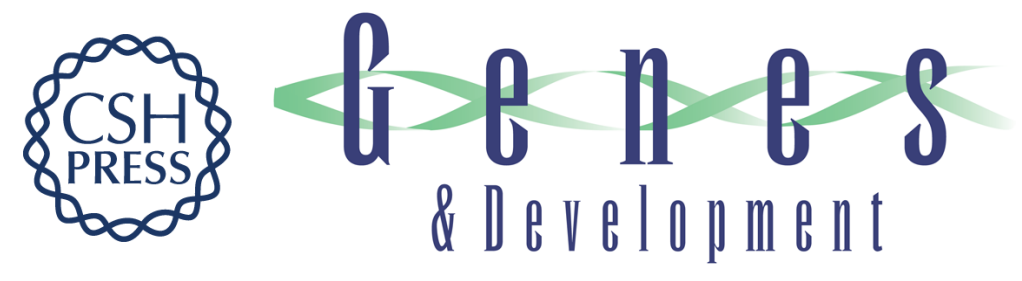

\section{The deficiency of Akt1 is sufficient to suppress tumor development in Pten $^{+/-}$mice}

Mei-Ling Chen, Pei-Zhang Xu, Xiao Peng, et al.

Genes Dev. 2006, 20:

Access the most recent version at doi:10.1101/gad.1395006

\section{Supplemental http://genesdev.cshlp.org/content/suppl/2006/06/01/20.12.1569.DC1 Material}

References This article cites 17 articles, 7 of which can be accessed free at: http://genesdev.cshlp.org/content/20/12/1569.full.html\#ref-list-1

\section{License}

Email Alerting

Receive free email alerts when new articles cite this article - sign up in the box at the top Service 